\title{
高阶代数微分方程的单值亚纯解 和有限多分支解
}

\author{
何育赞萧修治 \\ （中国科学院数学所, 北京）（武汉大学数学䒺)
}

\section{摘要}

本文应用 Nevanlinna 值分布理论, 讨论了次之一般高阶代数微分方程在复域中 大范围单值亚纯解和有限多分支解的存在性定理,

$$
\sum_{(i)} a_{(i)}(z)(u)^{i_{0}} \cdots\left(u^{(n)}\right)^{i_{n}}=\sum_{i=1}^{k} a_{i}(z) u^{i} / \sum_{j=0}^{l} b_{j}(z) u^{i},
$$

其中 $\left\{a_{i j}(z)\right\},\left\{a_{i}(z)\right\}$ 和 $\{b,(z)\}$ 为亚纯函数,获得精确形式的 Malmquist 型定理， 并且给出微分方程及其解的例说明定理中的界能被达到．最后得到一类代数微分方 程代数体函数解的增长性估计.

\section{一、引 言}

一阶常微分方程大范围单值亚纯解和有限多值解析解, 即代数体函数解的存在性问题, 首先由 Malmquist ${ }^{[1]}$ 所研究, 他所得到的重要定理现已以其名而著称. 吉田耕作 ${ }^{[2,3]}$ 首先应用 Nevanlinna 值分布理论的方法重新证明, 并推广了 Malmquist 定理, 其后 Valiron, Wittich 和 Lane 等人 ${ }^{[4-10]}$ 用不同的方法研究了某些类型方程的 Malmquist 定理. 本文讨论了一般的高 阶代数微分方程大范围单值亚纯解和有限多分支解的存在性定理, 获得精确形式的 Malmquist 型定理,以及解的增长性等.

我们考虑次之微分方程

$$
Q\left(u^{(n)}, u^{(n-1)}, \cdots, u, z\right)=R(u, z),
$$

其中 $Q(u) \equiv Q\left(u^{(n)}, u^{(n-1)}, \cdots, u, z\right) \equiv \sum a_{i_{0} \cdots i_{n}} z(u)^{i_{0}}\left(u^{\prime}\right)^{i_{1}} \cdots\left(u^{(n)}\right)^{i_{n}}$ 是微分多项式，

$$
R(u, z) \equiv P_{k}(u, z) / P_{l}(u, z) \equiv \sum_{i=0}^{k} a_{i}(z) u^{i} / \sum_{j=0}^{l} b_{j}(z) u^{i},
$$

系数 $\left\{a_{(i)}(z) \equiv a_{i_{0} \cdots i_{n}}(z)\right\},\left\{a_{i}(z)\right\}$ 和 $\left\{b_{j}(z)\right\}$ 为 $z$ 的亚纯函数或有理函数.

定义 1. 设 $u(z)$ 为 $\nu$ 值代数体函数, 除去一总长为有限的 $r$ 的区间序列, $s(r, u)$ 是满 足条件

$$
\lim _{r \rightarrow \infty} \frac{S\left(r, \frac{u}{}\right)}{T(r, u)}=0
$$

本文 1982 年 1 月 20 日收到。 
的 $r$ 的正实值函数, 若

$$
\sum_{(i)} T\left(r, a_{(i)}+\sum_{i=0}^{k} T\left(r, a_{i}\right)+\sum_{i=0}^{l} T\left(r, b_{j}\right)=S(r, u),\right.
$$

则称 $u(z)$ 对 $\left\{a_{(i)}(z)\right\},\left\{a_{i}(z)\right\}$ 和 $\left\{b_{i}(z)\right\}$ 是可允许函数. 方程 (1) 满足条件 (3) 的解称为 可允许解.

定义 2. 微分多项式 $Q(u)$ 的通项为 $a_{(i)} u^{i_{0}} \cdot\left(u^{\prime}\right)^{i} \cdots\left(u^{(n)}\right)^{i_{n}}$ ，设

$$
\left.\begin{array}{l}
\lambda_{(i)}=i_{0}+i_{1}+\cdots+i_{n}, \\
\boldsymbol{\mu}_{(i)}=i_{0}+2 i_{1}+\cdots+(n+1) i_{n}, \\
\vec{\mu}_{(i)}=i_{1}+2 i_{2}+\cdots+n i_{n}, \\
\sigma_{(i)}=i_{1}+3 i_{2}+\cdots+(2 n-1) i_{n} .
\end{array}\right\}
$$

令

$$
\left.\begin{array}{l}
\lambda=\max \left\{\lambda_{(i)}\right\}, \\
\mu=\max \left\{\mu_{(i)}\right\}, \\
\bar{\mu}=\max \left\{\vec{\mu}_{(i)}\right\}, \\
\sigma=\max \left\{\sigma_{(i)}\right\} .
\end{array}\right\}
$$

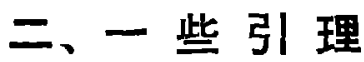

引理 1. 设 $P_{k}(u, z) \equiv a_{0}(z)+a_{1}(z) u+\cdots+a_{k}(z) u^{k}$, 又设 $Q_{1}(u) \equiv \sum a_{i_{0} \cdots i_{n}}(z) u^{i_{0}} \ldots$ $\left(u^{(n)}\right)^{i_{n}}$ 和 $Q_{2}(u) \equiv \Sigma b_{i_{0} \cdots / m}(z) u^{i_{0}} . \cdot\left(u^{(m)}\right)^{j_{m}}$ 为两个微分多项式. 若 $u=u(z)$ 为 $\nu$ 值代数体 函数, 它对于 $P_{k}(u, z), Q_{1}(u)$ 和 $Q_{2}(u)$ 的系数 $\left\{a_{i}(z)\right\},\left\{a_{(i)}(z)\right\}$ 和 $\left\{b_{(j)}(z)\right\}$ 是可允许函数, 且满足

$$
P_{k}(u, z) Q_{1}(u)=Q_{2}(u),
$$

则当 $k \geqslant \lambda^{\prime}$ 时, 有

$$
m\left(r, Q_{1}\right)=S(r, u),
$$

其中 $\lambda^{\prime}=\operatorname{mix}\left\{\lambda_{(j)}^{\prime}\right\}, \lambda_{(j)}^{\prime}=i_{0}+\cdots+i_{m}$. 更且在 $\nu=1$ 的情形, 当 $k>\mu^{\prime}$ 时,有

$$
T\left(r, Q_{1}\right)=S(r, u) \text {. }
$$

证. 设 $Q_{1}(u)$ 的通项为:

$$
F_{(i)}(z) \equiv a_{(i)}(z) u^{i_{0}} \cdots\left(u^{(n)}\right)^{i n} .
$$

今设 $u_{l}(z)(j=1,2, \cdots, v)$ 为 $u(z)$ 的一个分支,并记

$$
F_{(i) j}(z) \equiv a_{(u)}(z)\left(u_{j}(z)\right)^{i_{0}} \cdot \cdot\left(u_{j}^{(n)}(z)\right)^{i_{n}} \text {. }
$$

令 $E_{r}=\{z:|z|=r\}, E_{1}^{j}=\left\{z \in E_{r} ;\left|u_{j}(z)\right|<1\right\}$ 和 $E_{2}^{j}=E_{r} \backslash E_{1}^{j}$. 于是在 $E_{1}^{j}$ 上, 我们 有

$$
\left|F_{(i) j}(z)\right| \leqslant\left|a_{(i)}(z)\right|\left|\frac{u_{j}^{\prime}(z)}{u_{j}(z)}\right|^{i_{1}} \cdots\left|\frac{u_{j}^{(n)}(z)}{u_{j}(z)}\right|^{i_{n}}
$$

从而可得

$$
\frac{1}{2 x} \int_{E_{1}^{j}} \ln +\left|F_{(i) i}(z)\right| d \varphi \leqslant \frac{1}{2 \pi} \int_{E_{1}^{j}} \ln ^{+}\left|a_{(i)}(z)\right| d \varphi
$$




$$
+\frac{1}{2 \pi} \sum_{a=1}^{n} i_{\alpha} \int_{E_{1}^{j}} \ln +\left|\frac{u_{j}^{(\alpha)}(z)}{u_{j}(z)}\right| d \varphi,
$$

其中 $z=r e^{i \Phi}$, 进而我们有

$$
\begin{aligned}
& \frac{1}{2 \pi} \int_{E_{1}^{j}} \ln ^{+}\left|Q_{1}\left(u_{j}(z)\right)\right| d \varphi \leqslant \frac{1}{2 \pi} \sum_{(i)} \int_{L_{1}^{j}} \ln ^{+}\left|F_{(i) j}(z)\right| d \varphi+O(1) \\
& \leqslant \frac{1}{2 \pi} \sum_{(r)} \int_{E_{1}^{j}} \ln ^{+}\left|a_{(i)}(z)\right| d \varphi+\frac{1}{2 \pi} \sum_{(i)} \sum_{a=1}^{n} i_{\alpha} \int_{E_{1}^{j}} \ln +\left|\frac{u_{j}^{(\alpha)}(z)}{u_{j}(z)}\right| d \varphi+O(1) .
\end{aligned}
$$

在 $E_{2}^{j}$ 上我们将作如下的讨论. 令

$$
P_{k}^{j}(z)=P_{k}\left(u_{i}(z), z\right)=a_{k}(z)\left\{u_{j}^{k}(z)+A_{1}(z) u_{j}^{k-1}(z)+\cdots+A_{k}(z)\right\},
$$

其中 $A_{1}(z)=a_{k-1}(z) / a_{k}(z), \cdots, A_{k}(z)=a_{0}(z) / a_{k}(z)$. 对任一复数 $z$, 我们定义

$$
A(z)=\max \left\{1,\left|A_{1}(z)\right|, \cdots,\left|A_{k}(z)\right|^{\frac{1}{k}}\right\},
$$

并命 $E^{j}=\left\{z \in E_{r},\left|u_{j}(z)\right| \leqslant 2 A(z)\right\}$, 再命 $E_{21}^{j}=E_{2}^{j} \cap E^{i}$ 和 $E_{22}^{j}=E_{2}^{j} / E_{21}^{j}$. 于是 $E_{2}^{j}=$ $E_{21}^{j} \cup E_{22}^{j}$, 现分别在 $E_{21}^{j}$ 和 $E_{22}^{j}$ 上讨论. 在 $E_{21}^{j}$ 上注意到 $\left|u_{j}(z)\right| \leqslant 2 A(z)$, 我们存

$$
\begin{aligned}
\left|F_{(i) j}(z)\right| & =\left|a_{(i)}(z)\right|\left|u_{j}(z)\right|^{i_{0}+i_{1}+\cdots+i_{n}}\left|\frac{u_{j}^{\prime}(z)}{u_{j}(z)}\right|^{i_{1}} \ldots\left|\frac{u_{j}^{(n)}(z)}{u_{j}(z)}\right|^{i_{n}} \\
& \leqslant 2^{\lambda_{1}}(A(z))^{\lambda_{(i)}}\left|a_{(i)}(z)\right|\left|\frac{u_{j}^{\prime}(z)}{u_{j}(z)}\right|^{i_{1}} \ldots\left|\frac{u_{j}^{(n)}(z)}{u_{j}(z)}\right|^{i_{n}} .
\end{aligned}
$$

于是再有

$$
\begin{aligned}
& \frac{1}{2 \pi} \int_{F_{21}^{j}} \ln +\mid Q_{1}\left(u,(z)\left|d \varphi \leqslant \frac{1}{2 \pi} \sum_{(i)} \int_{E_{21}^{j}} \ln ^{+}\right| a_{(i)}(z) \mid d \varphi+\frac{\lambda_{(z)}}{2 \pi} \int_{E_{21}^{i}} \ln ^{+} A(z) d \varphi\right. \\
& +\frac{1}{2 \pi} \sum_{(i)} \sum_{a=1}^{n} i_{a} \int_{E_{21}^{j}} \ln ^{+}\left|\frac{u_{j}^{(\alpha)}(z)}{u_{j}(z)}\right| d \varphi+O(1) .
\end{aligned}
$$

在 $E_{22}^{j}$ 上, 首先有

$$
\begin{aligned}
\left|P_{k}^{j}(z)\right| & =\left.\left|a_{k}(z)\right| u_{j}(z)\right|^{k}\left|1+A_{1}(z) u_{j}^{-1}+\cdots+A_{k}(z) u_{j}^{-k}\right| \\
& \geqslant\left|a_{k}(z)\right|\left|u_{j}(z)\right|^{k}\left\{1-\frac{\left|A_{1}(z)\right|}{\left|u_{j}\right|}-\cdots-\frac{\left|A_{k}(z)\right|}{\left|u_{j}\right|^{k}}\right\} \\
& \geqslant\left|a_{k}(z)\right|\left|u_{1}\right|^{k}\left\{1-\frac{A(z)}{2 A(z)}-\cdots-\left(\frac{A(z)}{2 A(z)}\right)^{k}\right\} \\
& =\left|a_{k}(z)\right|\left|u_{i}\right|^{k} / 2^{k+1} .
\end{aligned}
$$

于是由方程 (6) 知, 在 $E_{22}^{i}$ 上

$$
\left|Q_{1}\left(u_{j}(z)\right)\right|=\left|\frac{Q_{2}(u,(z))}{P_{k}^{\prime}(z)}\right| \leqslant\left.\frac{2^{k+1}}{\left|a_{k}(z)\right|}\left|\sum_{(j)}\right| b_{(j)}(z)|| u_{j}\right|^{\left.\lambda_{j}\right)^{-k}}\left|\frac{u_{j}^{\prime}}{u_{j}}\right|^{z_{1}} \ldots\left|\frac{u_{j}^{(m)}}{u_{j}}\right|^{i_{m}} .
$$

但由假设 $k \geqslant \lambda^{\prime} \geqslant \lambda_{(j)}$, 并且在 $E_{22}^{j}$ 上 $\left|u_{j}(z)\right| \geqslant 1$, 上式成为:

$$
\left|\Omega_{1}\left(u_{j}(z)\right)\right| \leqslant \frac{2^{k+1}}{\left|a_{k}(z)\right|} \sum_{(j)}\left|b_{(j)}(z)\right|\left|\frac{u_{i}^{\prime}}{u_{j}}\right|^{i_{1}} \cdots\left|\frac{u_{j}^{(m)}}{u_{j}}\right|^{i_{m}},
$$

于是

$$
\frac{1}{2 \pi} \int_{E_{22}^{j}} \ln +\left|Q_{1}\left(u_{j}(z)\right)\right| d \varphi \leqslant \frac{1}{2 \pi} \int_{E_{22}^{j}} \ln ^{+}\left|\frac{1}{a_{k}(z)}\right| d \varphi+\frac{1}{2 \pi} \sum_{(j)} \int_{E_{22}^{j}} \ln { }^{+}\left|b_{(j)}(z)\right| d \varphi
$$




$$
+\frac{1}{2 \pi} \sum_{\beta=1}^{m} i_{\beta} \int_{F_{22}^{j}} \ln +\left|\frac{u_{j}^{(\beta)}}{u_{j}}\right| d \varphi+O(1) .
$$

综上诸式, 又根据引理中关于 $u(\approx)$ 为可允许解的假设和代数作函数对数导数的基本引理, 最 店得到

$$
\begin{aligned}
m\left(r, Q_{1}\right) & =\frac{1}{2 \pi \nu} \sum_{j=1}^{\nu} \int_{F_{r}} \ln +\left|Q_{1}(u,(z))\right| d \varphi \\
& =\frac{1}{2 \pi \nu} \sum\left\{\int_{E_{21}^{j}}+\int_{E_{22}^{j}}+\int_{E_{21}^{i}}\right\} \ln +\left|\Omega_{1}\left(u_{1}^{\prime}(z)\right)\right| d \varphi \\
& =S(r, u),
\end{aligned}
$$

即得 (7) 式.

当 $v=1$ 时, 我们将要证明 (8) 式成立, 为此我们考虑 $\Omega_{1}(u(z)) \equiv \Sigma a_{(i)}(z)(u(z))^{i_{0}} \ldots$ $\left(u^{(n)}(z)\right)^{n}$ 之极点, 这些极点可能出现为系数 $\left\{a_{(i)}(z)\right\}$ 之极点, 或 $u(z)$ 及其导数 $u^{\prime}(z), \cdots$, $u^{(n)}(z)$ 之极点. 下面我们将断言 $\Omega_{1}(u(z))$ 之极点, 它由 $u(z)$ 及其导数之极点产生者必包含 于系数 $\left\{a_{(i)}(z)\right\},\left\{a_{i}(z)\right\}$ 之零点和 $\left\{b_{(j)}(z)\right\}$ 之极点之中. 事实上, 若 $z_{0}$ 是 $u(z)$ 的 $p$ 重极 点, 但非为上述系数的零点和极点者, 则 $z_{0}$ 是 (6) 式左端之极点其重级至少为 $k p$, 另一方面 $z_{0}$ 是 (6) 式右端之极点其重级至多为 $\mu^{\prime} p$, 这就导出 $k \leqq \mu^{\prime}$, 这与所设矛盾. 现在我们进一 步证明由 $u(z)$ 之极点产生的 $Q_{1}(u(z))$ 的极点在 $N\left(r, Q_{1}(u)\right)$ 中贡献为 $S(r, u)$. 事实上, 若 $z_{0}$ 是 $u(z)$ 的 $p$ 重极点, 它同时是某个 $b_{(j)}(z)$ 的 $\tau_{(j)}$ 重极点和某个 $a_{1}(z)$ 的 $\tau_{i}$ 重零点, 则由表 达式 $Q_{1}(u(z))=Q_{2}(u(z)) / P_{k}(u(z), z)$ 知, $z_{0}$ 至多是分子 $Q_{2}(u(z), z)$ 的 $\mu^{\prime} p+\sum_{(j)} \tau_{(j)}$ 重极 点, 并且当 $k p-\sum_{i} \tau_{i}>0$ 时, 它至少是分母 $P_{k}(u(z), z)$ 的 $k p-\sum_{i} \tau_{i}$ 重极点, 因而计及 $\mu^{\prime}<k$, 我们有 $Q_{1}(u(z))$ 以 $z_{0}$ 为极点其重级至多是 $\mu^{\prime} p+\sum_{(i)} \tau_{(j)}-\left(k p-\sum_{i} \tau_{i}\right) \leqq \Sigma \tau_{(j)}+$ $\Sigma \tau_{i}$; 当 $k p-\sum_{i} \tau_{i} \leqq 0$ 时, 有 $p \leqq \frac{1}{k} \sum_{i} \tau_{i}$, 这说明 $z_{0}$ 作为 $u(z)$ 之极点其重级能由系数 $\left\{a_{i}(z)\right\}$ 以 $z_{0}$ 为零点的重级所界图, 因而对 $Q_{1}(u(z))$ 亦然. 因此两种情形皆有由 $u(z)$ 之极 点产生的 $Q_{1}(u(z))$ 之极点在 $|z|<r$ 内至多为 $\Sigma n\left(r, b_{(j)}\right)+\Sigma n\left(r, \frac{1}{a_{i}}\right)$. 注意到 $Q_{1}(u(z))$ 之极点, 还能由 $\left\{a_{(i)}(z)\right\}$ 之极点和 $u(z)$ 的导数 $u^{\prime}(z), \cdots, u^{(n)}(z)$ 之极点所产生, 于是 $Q_{1}(u(z))$ 在 $|z|<r$ 内的极点数有如下的估计

$$
n\left(r, Q_{1}(u)\right) \leqq \lambda\left\{\Sigma\left[n\left(r, a_{(i)}\right)+n\left(r, \frac{1}{a_{(i)}}\right)\right]+\sum n\left(r, b_{(j)}\right)+\Sigma n\left(r, \frac{1}{a_{i}}\right)\right\},
$$

由此即得

$$
N\left(r, Q_{1}(u)\right)=S(r, u),
$$

结合 (7) 和 $\left(7^{\prime}\right)$ 式便得 (8) 式.

引理 2. 设 $u(z)$ 为 $\nu$ 值代数体函数, 它对于 $P_{l}(u, z) \equiv b_{0}(z)+b_{1}(z) u+\cdots+b_{l}(z) u$ 的系数 $\left\{b_{i}(z)\right\}$ 是可允许函数,则有

$$
l T(r, u)-S(r, u) \leqslant T\left(r, P_{l}(u(z), z)\right) \leqslant l T(r, u)+S(r, u) .
$$

证. 我们在文献 [9] 中曾证明 


$$
l N(r, u)-S(r, u) \leqslant N\left(r, P_{l}(u, z)\right) \leqslant l N(r, u)+S(r, u) .
$$

又仿文献 [7] 类似引理 1 一样演算, 可得

$$
\ln (r, u)-S(r, u) \leqslant m\left(r, P_{l}(u, z)\right) \leqslant \operatorname{lm}(r, u)+S(r, u),
$$

结合上述两式即得 (9) 式.

引理 3. 设 $Q_{1}(u)$ 为引理 1 所述的微分多项式, $u(z)$ 为 $\nu$ 值代数体函数, 它对于 $Q_{1}(u)$ 的 系数 $\left\{a_{(i)}(z)\right\}$ 是可允许函数,则有

$$
m\left(r, g_{1}\right) \leqslant \lambda m(r, u)+S(r, u),
$$

并且

$$
N\left(r, \Omega_{1}\right) \leqslant \lambda N(r, u)+\bar{u} \bar{N}(r, u)+2(\nu-1) \sigma T(r, u)+S(r, u),
$$

其中 $\lambda, \bar{\mu}, \sigma$ 是前面所定义的数.

证. 如引理 1 一样演算, 设 $u_{j}(z)(j=1,2, \cdots, \nu)$ 为 $u(z)$ 的一个分支, 则有

$$
\Omega_{1}\left(u_{j}(z)\right)=\sum_{(i)} a_{(i)}(z) u_{i}^{l}(i)(z)\left(\frac{u_{i}^{\prime}(z)}{u_{i}(z)}\right)^{i_{1}} \cdots\left(\frac{u_{i}^{(n)}(z)}{u_{i}(z)}\right)^{i_{n}},
$$

于是

$$
\begin{aligned}
m\left(r, Q_{1}\right)= & -\frac{1}{2 \pi \nu} \sum_{j=1}^{\nu} \int_{E_{r}} \ln +\left|Q_{1}(u,(z))\right| d \varphi \\
\leqslant & \frac{1}{2 \pi \nu} \sum_{j=1}^{\nu}\left\{\sum_{(i)} \int_{E_{r}} \ln ^{+}\left|a_{(i)}(z)\right| d \varphi+\lambda_{(i)} \int_{E_{r}} \ln +\left|u_{1}(z)\right| d \varphi\right. \\
& \left.+\sum_{a=1}^{n} i_{a} \int_{E_{r}} \ln +\left|\frac{u_{j}^{(\alpha)}(z)}{u_{l}(z)}\right| d \varphi\right\}+O(1) \\
\leqslant & \lambda m(r, u)+S(r, u) .
\end{aligned}
$$

为了计算 $Q_{1}(u(z))$ 之极点数, 设通项为:

$$
F_{(i)}(z) \equiv a_{(i)}(z) u^{i_{0}}(z) \cdots\left(u^{(n)}(z)\right)^{i},
$$

于是

$$
n\left(r, F_{(i)}\right) \leqslant n\left(r, a_{(i)}\right)+i_{0} n(r, u)+\cdots+i_{n} n\left(r, u^{(n)}\right) .
$$

但知对任意 $\alpha$, 有 ${ }^{[9]}$

$$
n\left(r, u^{(\alpha)}\right) \leqslant n(r, u)+\alpha \bar{n}(r, u)+(2 \alpha-1) n_{x}(r, u),
$$

其中 $n_{x}(r, u)$ 表示 $|z| \leqslant r$ 内分支点个数, 于是

$$
n\left(r, F_{(i)}\right) \leqslant n\left(r, a_{(i)}\right)+\lambda_{(i)} n(r, u)+\vec{\mu}_{(i)} \bar{n}(r, u)+\sigma_{(i)} n_{x}(r, u) .
$$

今若 $z_{0}$ 为 $Q_{1}(u(z))$ 之极点, 则它必出现为 $Q_{1}(u(z))$ 的某些项之极点, 其重级至多是相加项中 以 $z_{0}$ 为极点的重级最高者. 换言之,

$$
n\left(r, Q_{1}\right) \leqslant \sum_{(i)} n\left(r, a_{(i)}\right)+\lambda n(r, u)+\bar{\mu} \vec{n}(r, u)+\sigma n_{x}(r, u) .
$$

从而得

$$
N\left(r, Q_{1}\right) \leqslant \lambda N(r, u)+\bar{\mu} \bar{N}(r, u)+\sigma N_{x}(r, u)+S(r, u),
$$

但知

$$
N_{x}(r, u) \leqslant 2(\nu-1) T(r, u)+O(1),
$$

从而即得 (11) 式. 
下面的引理当 $v=1$ 时为 A. Mохонькo ${ }^{[1]}$ 所证,一般情形亦成立 ${ }^{[10]}$.

引理 4. 设 $R(u, z) \equiv P_{k}(u, z) / P_{l}(u, z), P_{k}(u, z)=\sum_{i=0}^{k} a_{i}(z) u^{i}, P_{l}(u, z) \equiv \sum_{j=0}^{i} b_{l}(z) u^{j}$, 其中系数 $\left\{a_{i}(z)\right\},\left\{b_{l}(z)\right\}$ 为立纯函数, 若 $u(z)$ 为 $\nu$ 值代数体函数, 且对 $\{a,(z)\}$ 和 $\left\{b_{i}(z)\right\}$ 是可允许的, 则有

$$
T(r, R(u, z))=s T(r, u)+S(r, u),
$$

其中 $s=\max \{k, l\}$.

\section{三、主要结果}

本节中我们将证明高阶代数微分方程单值亚纯解和代数体函数解的 Malmquist 型定理, 并给出一微分方程及其解的例来说明定理中的界能被达到

定理 1. 若方程 (1) 存在 $\nu$ 值可允许解,则必

$$
s \leqslant \lambda+\bar{\mu}(1-\theta(u, \infty))+2(\nu-1) \sigma,
$$

其中 $s=\max \{k, l\}, \lambda, \pi$ 和 $\sigma$ 是 (5) 式中所定义的数 $\theta(u, \infty)=1-\overline{\lim } \frac{\bar{N}(r, a)}{T(r, u)}$.

证. 设 $u(z)$ 为方程 (1) 的 $v$ 值可允许代数体函数解, 由引理 4 得到

$$
T(r, R(u, z))=s T(r, u)+S(r, u),
$$

其中 $s=\max \{k, l\}$. 另一方面, 根据引理 3 有

$$
T(r, Q(u)) \leqslant[\lambda+2(\nu-1) \sigma] T(r, u)+\bar{\mu} \bar{N}(r, u)+S(r, u) .
$$

由假设 $u(z)$ 为方程 (1) 的解, 从而有

$$
\begin{aligned}
s T(r, u)+S(r, u) & =T(r, R(u, z))=T(r, Q(u)) \\
& \leqslant[\lambda+2(v-1) \sigma] T(r, u)+\bar{u} \bar{N}(r, u)+S(r, u) .
\end{aligned}
$$

注意到 $\theta(u, \infty)$ 的定义,由上式即得 (12) 式.

推论 1. 设 $u(z)$ 为方程 (1) 的可允许 $v$ 值代数体函数解, 若 $\theta(u, \infty)=1$, 则

$$
s \leqslant \lambda+2(\nu-1) \sigma,
$$

事实上, 当 $\theta(u, \infty)=1$ 时, (12) 式即化为上式.

推论 2. 设 $u(z)$ 为方程 (1) 的 $v$ 值可允许代数体函数解, 若 $\lambda+2(\nu-1) \sigma<s$. 则

$$
\theta(u, \infty) \leqslant 1-\frac{s-[\lambda+2(\nu-1) \sigma]}{\bar{\mu}} .
$$

特别地, 若 $s=\lambda+\bar{\mu}+2(\nu-1) \sigma$, 则必 $\theta(u, \infty)=0$.

证. 在定理的证明中, 我们有

$$
\{s-[\lambda+2(v-1) \sigma]\} T(r, u) \leqslant \bar{\mu} \bar{N}(r, u)+S(r, u) .
$$

于是

$$
\theta(u, \infty)=1-\varlimsup_{r \rightarrow \infty} \frac{\bar{N}(r, u)}{T(r, u)} \leqslant 1-\frac{s-(\lambda+2(\nu-1) \sigma)}{\bar{\mu}} .
$$

1. 设 $h(z)$ 为多项式, 则方程

$$
\frac{d u}{d z}=\frac{-u^{2 v}-h^{\prime}(z) u^{v}+h^{2}(z)}{\nu h(z) u^{\nu-1}}
$$


存在一个 $\nu$ 值可允许代数体函数解 $u(z)$, 它由下面的方程所定义:

$$
\cos z u^{\nu}+h(z) \sin z=0,
$$

且知 $\lambda=\vec{\mu}=\sigma=1, s-\max \{k=2 v, l=\nu-1\}=2 \nu$. 即有 $s=\lambda+\vec{\mu}+2(\nu-1) \sigma$, 从而 $\theta(u, \infty)=0$, 使定理的界被达到, 换言之我们的定理是精确的.

定理 2. 若方程（1）存在单值亚纯可允许解,则必 $R(u, z)$ 退化为 $u$ 的多项式, 且其次数

$$
k \leqslant \lambda+\vec{\mu}(1-\theta(u, \infty)) \text {. }
$$

证. 设

$$
R(u, z)=P_{k}(u, z) / P_{l}(u, z)=P_{k_{1}}(u, z)+P_{k_{2}}(u, z) / P_{l}(u, z),
$$

其中 $k_{2}<l$. 下面我们将首先证明在定理的条件下必有 $P_{k_{2}}(u, z) \equiv 0$. 事实上, 如若不然, 方程 (1) 改为:

或

$$
P_{l}(u, z)\left(Q(u)-P_{k_{1}}(u, z)\right)=P_{k_{2}}(u, z),
$$

$$
P_{l}(u, z) Q_{1}(u)=P_{k_{2}}(u, z),
$$

其中 $Q_{1}(u) \equiv Q(u)-P_{k_{1}}(u, z)$. 今若方程 (1) 存在可允许解 $u(z)$, 则亦方程为 $\left(1^{\prime}\right)$ 的可 允许解. 此时在引理 1 中, 令 $Q_{2}(u)$ 为 $P_{k_{2}}(u, z)$, 从而 $\mu^{\prime}=k_{2}$, 但知 $l>k_{2}$, 于是

$$
T\left(r, Q_{1}(u)\right)=S(r, u) \text {, }
$$

由方程 (1') 可知

$$
\begin{aligned}
T\left(r, P_{l}(u, z)\right) & \leqslant T\left(r, P_{k_{2}}(u, z)\right)+T\left(r, \frac{1}{Q_{1}(u)}\right) \\
& \leqslant T\left(r, P_{k_{2}}(u, z)\right)+S(r, u)
\end{aligned}
$$

另外, 根据引理 2 , 我们有

$$
\begin{aligned}
l T(r, u)-S(r, u) & \leqslant T\left(r, P_{l}(u, z)\right) \leqslant T\left(r, P_{k_{2}}(u, z)\right)+S(r, u) \\
& \leqslant k_{2} T(r, u)+S(r, u) .
\end{aligned}
$$

上式两边除以 $T(r, u)$, 并令 $r \rightarrow \infty$, 则导出 $l \leqslant k_{2}$, 这与所设矛盾, 故必须 $P_{k_{2}}(u, z) \equiv 0$.

我们将进一步证明 (13) 式,由上述证明可知,若方程 (1) 存在可允许亚纯解，则必 $R(u$, $z)$ 退化为 $u$ 的多项式, 因而可设方程为

$$
Q(u)=P_{k}(u, z) \text {. }
$$

根据引理 3 , 当 $\nu=1$ 时, 我们得到

$$
m(r, Q(u)) \leqslant \lambda m(r, u)+S(r, u),
$$

和

$$
N(r, Q(u)) \leqslant \lambda N(r, u)+\bar{\mu} \bar{N}(r, u)+S(r, u) .
$$

将上述两式相加, 即得

$$
T(r, Q(u(z))) \leqslant \lambda T(r, u)+\bar{\mu} \bar{N}(r, u)+S(r, u),
$$

另外, 应用引理 2 有

$$
T(r, Q(u))=T\left(r, P_{k}(u, z)\right) \geqslant k T(r, u)-S(r, u),
$$

结合 (14) 和 (15) 式便得

$$
k T(r, u) \leqslant \lambda T(r, u)+\bar{\mu} \bar{N}(r, u)+S(r, u),
$$

由此立即导出 (13) 式, 定理 2 证毕. 
附注。本定理将文献 [10] 的结果精密化了, 并推广了文献 $[1,4-6]$ 的结果。若 $\theta(u$ ， $\infty)=0$ 即为文献 $\left[101\right.$ 之结果. 特别地: $Q(u) \equiv \frac{d u}{d z}$, 则为

$$
\frac{d u}{d z}=R(u, z),
$$

即得 Malmquist 在文献 [1] 中的基本定理的推广, 在那里 $R(u, z)$ 为其变元的有理函数,这里 对于 $z$ 是亚纯函数.

若 $\theta(u, \infty)=1$, 则 (13) 式成为 $k \leqslant \lambda$, 这包含了文献 [5, 6] 中的结果为其特殊情形, 在那里讨论了方程的可允许整函数解的情形.

下面我们将首次给出一类代数微分方程有限多分支解的增长性的估计.

设 $Q(u)=Q\left(u, u^{\prime}, \cdots, u^{(n)}, z\right)$ 为微分多项式, 其系数为 $z$ 的亚纯函数. 对非负整数 $q$, 设 $Q_{u}(u)$ 是 $Q(u)$ 中次数为 $q$ 的齐次部分. 对每个 $r>0$, 令

$$
\Delta(r)=\max \left\{T\left(r, a_{(i)}\right)\right\} .
$$

定理 3. 设 $u_{0}(z)$ 是不恒为零的, 满足方程 $Q(u)=0$ 的代数体函数解,但对某个非负整数 $q$, 它不满足方程 $Q_{q}(u)=0$, 则有

$$
T\left(Q, u_{0}\right) \leqslant K F(r),
$$

其中 $K$ 为一正的数值常数及

$$
F(r)=\bar{N}\left(r, u_{0}\right)+\bar{N}\left(r, \frac{1}{u_{0}}\right)+N_{x}\left(r, u_{0}\right)+S\left(r, u_{0}\right)+\Delta(r) .
$$

证. 设 $w_{0}=u_{0}^{\prime} / u_{0}$, 则由归纳法易知（参见文献 [13]）, 对每个 $i \geqslant 1, u_{0}^{(i)} / u_{0}$ 可写为非负 整系数的 $w_{0}, w_{0}^{\prime}, \cdots, w_{0}^{(j-1)}$ 的多项式. 因此, 对每个非负整数 $q, Q_{q}\left(u_{0}\right) /\left(u_{0}\right)^{q}$ 可写为 $::_{0}$, $w_{0}^{\prime}, \cdots, w_{0}^{(s-1)}$ 的多项式 $P_{q}\left(w_{0}, z\right)$, 其系数为 $Q_{q}$ 中系数的常系数线性组合. 若 $m$ 是 $u_{0}(z)$ 不 满足齐次方程 $Q_{q}(u)=0$ 之 $q$ 的最大值, 计及 $u_{0}$ 为 $Q(u)=0$ 的解,得到

$$
\sum_{q=0}^{m} P_{q}\left(w_{0}, w_{0}^{\prime}, \cdots, w_{0}^{(n-1)}, z\right)\left(u_{0}(z)\right)^{q} \equiv 0 \text {. }
$$

即

$$
P_{m}\left(w_{0}, z\right)\left(u_{0}(z)\right)^{m}=-\sum_{q=0}^{m-1} P_{q}\left(w_{0}, z\right) u_{0}^{q} .
$$

类似于引理 2 的证明,不难得到

$$
\begin{gathered}
m T\left(r, u_{0}\right) \leqslant T\left(r, P_{m} u_{n}^{m}\right)+T\left(r, P_{m}\right)+O(1), \\
T\left(r, \sum_{q=0}^{m-1} P_{q} u_{0}^{q}\right) \leqslant(m-1) T\left(r, u_{0}\right)+(m-1) \sum_{q=0}^{m-1} T\left(r, P_{q}\right)+O(1),
\end{gathered}
$$

因而得到

$$
T\left(r, u_{0}\right) \leqslant m \sum_{q=0}^{m} T\left(r, P_{q}\right)+O(1)
$$

而对于每个 $j \geqslant 1$

$$
T\left(r, w_{0}^{(j)}\right) \leqslant 2\left\{\bar{N}\left(r, u_{0}\right)+\bar{N}\left(r, \frac{1}{u_{0}}\right)+[(\nu-1)(2 j-1)+1] N_{x}\left(r, u_{0}\right)\right\}+S\left(r, u_{0}\right) .
$$


将上式代入 (19) 式,且注意到 $\Delta(r)$ 的定义,则完成了定理 3 的证明.

注. $u_{0}(z)$ 同时满足所有齐次方程 $Q_{u}(u)=0$ 的情形，我们将另作讨论，不再帮述.

作者事心感谢庄妡泰教授的宝贵意见和对本文的细心恕阅。

\section{考文若献}

[1] Malmquist, J., Aata Malli, 36(1913), 297-340̈.

[ 2] Yosida, K., Japan J. Math., 9(1933), 253-256.

[ 3 ] —. ibid., $10(1934), 1-20$.

[4] Laine, I., Ann. Acad. Sci. Fenn., Ser. AI, 487(1971), 22pp.

[5] Anastassiadis, J., Bull. Sci. Math., 76(1952), 57-64.

[6] Valiron, G., ibid., 76(1952), 144-148.

[ 7 ] 萧修治, 数学学报, 15 (1965), 397-405.

[ 8 ] Steinmetz, N., Dissertation, Karlruhe, 1978.

[9] 何育赞, 数学学报, 24(1981)，464-471.

[10] Gackstatter. F. und Laine I., Ann. Polonici Math., 38(1980), 259-287.

[11] Мохонько, А. З. И Мохонько, В. Д., СНБ, Мат. Ж., 15 (1974). 1305-1322.

[12] Bank, S. and Laine, I., Math. Scand., 40(1977), 119-126.

[13] Hiong, K. L., Sur les fonctions méromorphes et les fonctions algebrö̈des, Mémor. Sei. Math Parris, 1957, 101. 